\title{
Evaluación del riesgo cardiovascular en pacientes con infección por VIH en un centro de atención de salud especializado en Santiago de Chile
}

Camila Lazcano, Juan C. Millacura, Felipe Saavedra y Claudia P. Cortés

\section{Evaluation of cardiovascular risk in HIV positive patients in a specialized center at Santiago, Chile}

Antiretroviral therapy has changed the course of HIV epidemic, as consequence, the patients present the same medical conditions than the rest of the population, including cardiovascular diseases. Aim: To describe the evolution of cardiovascular risk of HIV positive patients attending to a HIV/AIDS integral clinical center. Clinical charts were reviewed, looking for cardiovascular risk markers. Our findings showed a deficient evaluation of the cardiovascular basal risks at first medical control and patients had important metabolic alterations despite hypolipidemic treatment. Given the higher cardiovascular risk of this population, increasing the effort on diagnosis and treatment of HIV patients is required.

Key words: HIV, cardio-vascular risk, hipolipidemic drugs.

Palabras clave: VIH, riesgo cardio-vascular, fármacos hipolipemiantes.

\section{Introducción}

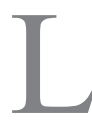

a incorporación de la terapia anti-retroviral (TARV) ha provocado un drástico cambio en la historia natural de la infección por VIH, en el mundo y en nuestro país ${ }^{1,2}$. Ha habido una disminución progresiva de la mortalidad a causa de enfermedades oportunistas, provocando un aumento en la expectativa de vida $^{3}$ y con ello el incremento de morbimortalidad por enfermedades crónicas, destacando entre ellas las patologías cardiovasculares $(\mathrm{CV})^{4}$.

Los factores que explican este fenómeno se pueden resumir en tres: la $\mathrm{TARV}^{5,6}$, la inflamación propia causada por el virus ${ }^{7}$ y la presencia de factores de riesgo $\mathrm{CV}$ clásicos, que en algunos casos, se encuentran aumentados en la población con infección por $\mathrm{VIH}^{8,9}$.

Este mayor riesgo CV observado en los pacientes con infección por VIH, es subestimado por las escalas de evaluación utilizadas comúnmente en la población general ${ }^{10}$.

El objetivo de nuestro estudio fue describir la pesquisa y seguimiento del riesgo $\mathrm{CV}$ al ingreso y en los controles sucesivos en pacientes con infección por VIH, controlados en un centro de atención integral de VIH/SIDA.

Universidad de Chile. Facultad de Medicina (CL, JCM, FS, CC).

Fundación Arriarán, Santiago de Chile (CC).

Ninguno de los autores declara conflicto de interés.

Fuente de financiamiento: no hubo.

Recibido: 13 de agosto de 2015 / Aceptado: 5 de abril de 2016

Correspondencia a:

Claudia Cortés M.

cpcortesm@med.uchile.cl

\section{Materiales y Métodos}

Se realizó un estudio observacional retrospectivo. Se revisaron las fichas clínicas de pacientes activos, ingresados entre los años 1991 y 2011, en control ambulatorio en Fundación Arriarán, Santiago de Chile. Los controles se realizaron entre marzo y julio de 2012. Las fichas fueron seleccionadas al azar, siendo una muestra representativa de la población del centro.

Se registraron los datos demográficos, fecha de ingreso a control, inicio y tipo de TARV, presencia de factores de riesgo CV: tabaquismo, hipertensión arterial (HTA), diabetes mellitus tipo 2 (DM2), dislipidemia y obesidad. Se buscó el antecedente de uso de terapia hipolipemiante, perfil lipídico, glicemia, creatinina y presión arterial al ingreso, al inicio de la TARV y de los últimos cuatro controles. Se buscaron los datos antropométricos: peso, talla, circunferencia abdominal y de cadera.

\section{Resultados}

Se revisaron 200 fichas clínicas, correspondientes a 10\% del total de pacientes activos en control durante el período estudiado. Un 86,5\% correspondieron a hombres. La mediana de edad al ingreso a los controles fue de 35,5 años. La mediana de años en control al momento del estudio fue de 5,8 años. Un 97\% de los pacientes recibían TARV, con una mediana de duración en terapia de 5,3 años.

De los antecedentes médicos presentes al ingreso se reportó $4 \%$ con HTA, 3\% con DM2, y 3,5\% con dislipidemia. Un único paciente registró el antecedente de un accidente cerebro vascular y ningún paciente registró infarto agudo del miocardio. El antecedente de obesidad fue descrito al ingreso en $2,5 \%$ de los pacientes. Se encontró el dato de tabaquismo activo en 19\%; sin embargo, 35,5\% no tenían información registrada.

En cuanto a las medidas antropométricas, el registro del peso estuvo presente en $51 \%$ de los pacientes, la talla disponible en $5 \%$ y la circunferencia abdominal y de cadera fue registrada en $1,5 \%$.

Del total de pacientes; $33,5 \%$ usaban fármacos hipolipemiantes. Un $83,6 \%$ estaba recibiendo gemfibrozilo, $8,9 \%$ estaba con terapia combinada (gemfibrozilo-atorvastatina) y 7,4\% recibía atorvastina sola.

E1 98,5\% de los usuarios de fármacos hipolipemiantes se encontraban con TARV y $94,7 \%$ de los que no los recibían estaba con TARV. No se encontraron diferencias significativas en la distribución de fármacos antiretrovirales utilizados entre ambos grupos.

La evolución del perfil metabólico se observa en la Figura 1. Se obtuvo el registro en $67 \%$ de los pacientes. Destaca la diferencia entre los pacientes con y sin terapia hipolipemiante, desde su ingreso al último control, siendo significativamente mayores los valores en pacientes usuarios de hipolipemiantes, tanto en los valores de triglicéridos (Figura 2a) como de colesterol total (Figura 2b).

\section{Discusión}

En nuestro estudio encontramos una deficiente evaluación del riesgo $\mathrm{CV}$ al ingreso al centro.

La baja cuantía de determinaciones no nos permite encontrar valores de colesterol, triglicéridos, IMC, etc. que sean representativos del estado de los pacientes al inicio de los controles; siendo estas determinaciones fundamentales para el manejo correcto de los riesgos $\mathrm{CV}$. 

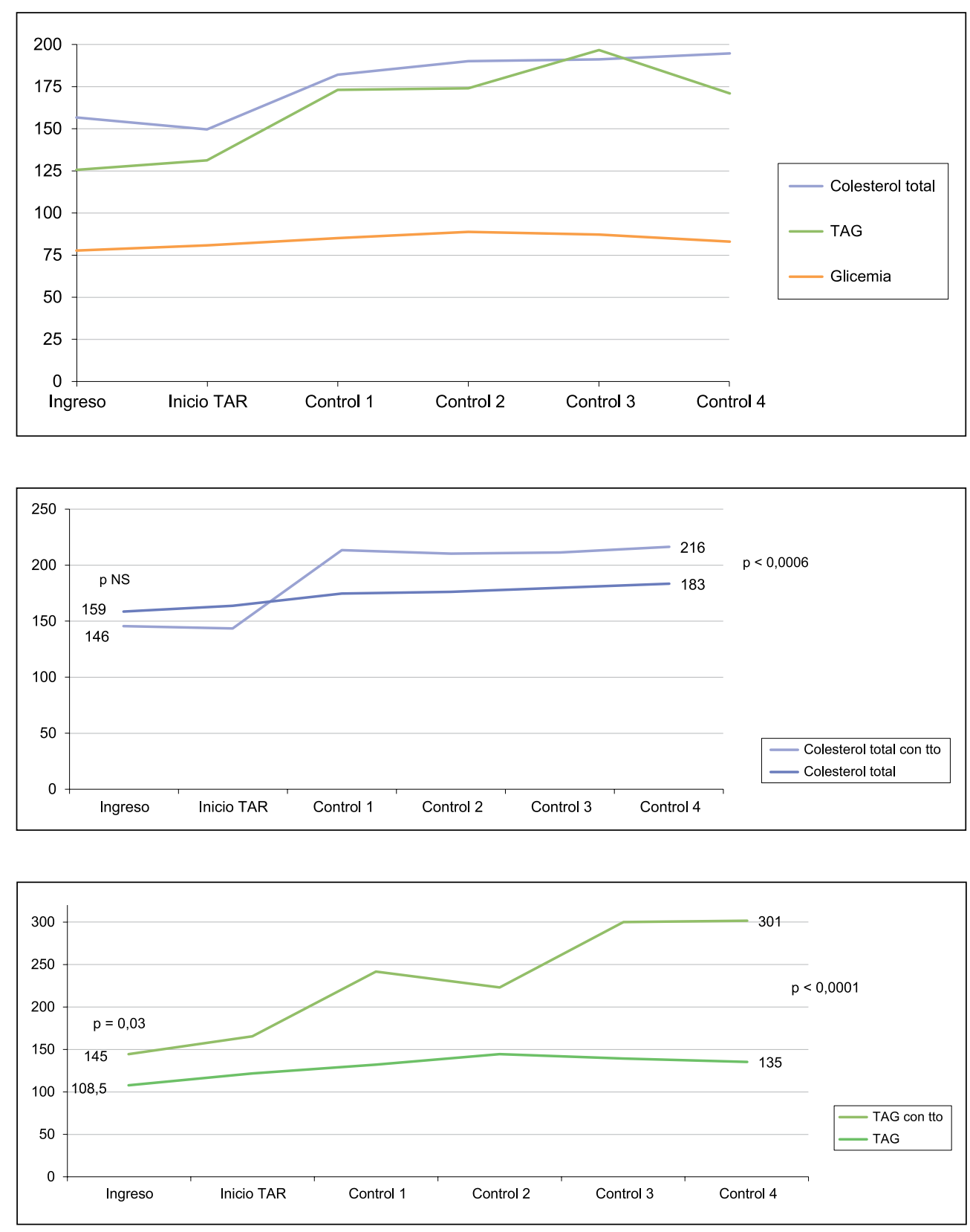

Figura 1. Evolución del perfil metabólico desde ingreso, inicio TARV y últimos cuatro controles.

Figura 2a. Evolución del colesterol total en pacientes con y sin terapia hipolipemiante al ingreso, inicio TARV y últimos cuatro controles.
Figura 2b. Evolución de triglicéridos (TAG) en pacientes con y sin terapia hipolipemiante, al ingreso, inicio TARV y últimos cuatro controles.
También es relevante considerar el aumento progresivo en los valores de los parámetros del perfil lipídico, sobre todo en las concentraciones de triglicéridos, que fue significativamente mayor en los pacientes que se encontraban en terapia hipolipemiante. Esta situación deja entrever un manejo insuficiente de los trastornos metabólicos, limitándose a la indicación de sólo un tratamiento farmacológico y no a una terapia integral como lo recomiendan las guías internacionales.

Es perentorio realizar una pesquisa precoz de los factores de riesgo CV en todos los pacientes, tanto a su ingreso como en los controles sucesivos, enfatizando en la prevención de los factores modificables y promoviendo la indicación de medidas no farmacológicas.
A la luz de nuestros resultados, es posible plantear la necesidad de integrar los controles médicos con un equipo multidisciplinario, de nutricionistas, médicos infectólogos y especialistas en el manejo del riesgo CV para así lograr un óptimo manejo de estos pacientes.

\section{Resumen}

La terapia anti-retroviral (TARV), ha mejorado significativamente la expectativa de vida de los pacientes con infección por VIH, quienes presentan enfermedades similares al resto de la población, incluyendo las patologías 
cardiovasculares. El objetivo del estudio fue describir la evolución del riesgo cardiovascular en pacientes con infección por VIH controlados en un centro de atención integral de VIH/SIDA. Se revisaron las fichas clínicas de los pacientes en control buscando la presencia de factores de riesgo cardiovascular. Nuestros hallazgos muestran una deficiente evaluación al ingreso del riesgo cardiovascular basal con importantes alteraciones metabólicas pese a la terapia hipolipemiante. Dado el mayor riesgo cardiovascular de esta población, se requiere mejorar su pesquisa y tratamiento en los pacientes con infección por VIH.

\section{Referencias bibliográficas}

1.- Palella F J Jr, Delaney K M, Moorman A C, Loveless M O, Fuhrer J, Satten G A, et al. Declining morbidity and mortality among patients with advanced human immunodeficiency virus infection. HIV Out patient Study Investigators. N Engl J Med 1998; 338: 853-60.

2.- Wolff M, Bustamante C, Bidart M T, Dabanch J, Diomedi P, Northland R. Impacto de la terapia anti retroviral en la mortalidad de pacientes VIH $(+)$ chilenos: estudio caso-control (MORTAR). Rev Med Chile 2000; 128: $839-45$.

3.- Hasse B, Ledergerber B, Furrer H, Battegay M, Hirschel B, Cavassini M, et al. Morbidity and aging in HIV-infected persons: the Swiss HIV cohort study. ClinInfect Dis 2011; 53: 1130-9.

4.- Gill J, May M, Lewden C, Saag M, Mugavero M, Reiss P, et al. Causes of death in HIV-1-infected patients treated with antiretroviral therapy, 1996-2006: collaborative analysis of 13 HIV cohort studies. Clin Infect Dis 2010; 50: 1387-96.

5.- Carr A, Samaras K, Thorisdottir A, Kaufmann G R, Chisholm D J, Cooper D A. Diagnosis, prediction, and natural course of HIV-1 proteaseinhibitor-associated lipodystrophy, hyperlipidaemia, and diabetes mellitus: a cohort study. Lancet 1999; 353: 2093-9.

6.- Durand M, Sheehy O, Baril J G, Lelorier J, Trembla C L. Association between HIV infection, antiretroviral therapy, and risk of acute myocardial infarction: a cohort and nested case-control study using Quebec's public health insurance data base. J Acquir Immune Defic Syndr 2011; 57: 245-53.

7.- Alonso-Villaverde C. Fisiopatología de la enfermedad cardiovascular en pacientes con VIH. Enferm Infecc Microbiol Clin 2009; 27 (Supl 1): 33-9.

8.- Niaura R, Shadel W G, Morrow K, Tashima K, Flanigan T, Abrams D B. Human immunodeficiency virus infection, AIDS, and smoking cessation: the time isnow. Clin Infect Dis 2000; 31: 808-12.

9.- Masia M, Pérez-Cachafeiro S, Leyes M, López Aldeguer J, López M, Segura D, et al. Riesgo cardiovascular en pacientes con infección por el virus de la inmunodeficiencia humana en España. Cohorte Co RIS. Enf Inf Microbiol Clin 2011; 30: 517-27.

10.- Estrada V, Bernardino J I, Masiá M, Iribarren J A, Ortega A, Lozano F, et al. Cardiovascular risk factors and lifetime risk estimation in HIV-infected patients under antiretroviral treatment in Spain. HIV Clin Trials 2015; 16: 57-65. 